Original Article

\title{
FIRST DETECTION OF NOSEMA CERANAE AND NOSEMA APIS IN GREATER WAX MOTH GALLERIA MELLONELLA
}

\author{
Erkay Ozgor ${ }^{1 *}$ \\ Irem Celebier ${ }^{2}$ \\ Meltem Ulusoy² \\ Nevin Keskin ${ }^{2}$ \\ 'Cyprus International University, Faculty of Arts and Sciences, 99258, Nicosia, \\ Mersin 10, Turkey \\ ${ }^{2}$ Hacettepe University, Department of Biology, 06800, Beytepe/Ankara, Turkey \\ *corresponding author: erkayozgor@gmail.com \\ Received: 16 March 2016; accepted: 4 June 2017
}

A b s tract

The greater wax moth Galleria mellonella L. (Lepidoptera: Pyralidae), one of the pests of honey bee (Apis mellifera L.) colonies, has spread almost all over the world. Although the G. mellonella is often reported to infest weak honey bee colonies that are exposed to pesticides and diseases, it is also a threat for healthy colonies. Therefore, there is a fairly high probability of transmission of both microflora-specific bacteria and pathogen microorganisms, especially Nosema species, between these organisms (Moth and bees). The aim of this study was to investigate the presence of Nosema species in greater wax moth G. mellonella collected from apiaries as well as grown in laboratory conditions. Adults and late instar larva of wax moth were used for detecting Nosema apis and Nosema ceranae. Real-time PCR amplification studies were performed and specific ITS regions were targeted to distinguish Nosema species. Real-time PCR results showed that $N$. apis and $N$. ceranae were found in both phases of $G$. mellonella. This is the first study to confirm that $\boldsymbol{N}$. apis and $\boldsymbol{N}$. ceranae are present in greater wax moth collected from apiaries and grown at laboratories in Turkey.

Keywords: Galleria mellonella, honey bee, Nosema apis, Nosema ceranae, real-time PCR

\section{INTRODUCTION}

The greater wax moth Galleria mellonella L. (Lepidoptera:Pyralidae) is an economic pest of honey bee (Apismellifera) colonies and nowadays has spread almost all over the world, including Europe, Asia, North America and Australia (Ellis, Graham \& Mortensen, 2013), especially spread to low altitude regions with a temperate climate (Akyol \& Korkmaz, 2008). The waxworms of the greater wax moth have been shown to be an excellent model organism for in vivo toxicology and pathogenicity testing, replacing the use of small mammals (Hu \& Webster, 2010; Coleman et al., 2011; Harding et al., 2013). In addition to the use of the wax moth as a model organism for research on insect physiology, genomics and proteomics, they have an important role in the apicultural industry (Ellis, Graham, \& Mortensen, 2013). The larval phase but not the adult phase causes severe damage to the combs and leads to significant economic loss in the beekeeping sector.

Female wax moths lay almost $0.5 \mathrm{~mm}$ eggs in crevices and cracks of the bee hive. Larvae emerge from the eggs within 5-8 days under normal conditions $\left(24-26^{\circ} \mathrm{C}\right)$ which varies depending on the temperature. Following development, the larvae weave a cocoon, transform into adults and complete their life cycle (Spangler, 1985; Williams, 1997; Ellis, Graham \& Mortensen, 2013. Besides their natural habitat, wax moths can be reared in laboratory conditions with the appropriate diet (Ellis, Graham, \& Mortensen, 2013).

The wax moth is often reported in honey bee colonies that are weakened by exposure to pesticides and diseases but is also a threat for healthy colonies. Its constantly interacts with honey bees because its essential nutrient is 
honeycomb which is inside bee nests (Ellis, Graham, \& Mortensen, 2013). Therefore, both microflora-specific bacteria and pathogen microorganisms, especially Nosema species, very likely are transferred between the moths and bees, which increases the probability of contact with honey bee pathogens and parasites. Wax moths mostly prefer weak and diseased honey bee colonies because of the easier access to nutrients. This situation increases the probability of contact with honey bee pathogens and parasites.

Besides reports on Nosema galleriae infection and lytic activity through the injection of Nosema algerae and Nosema plodiae (Kučera \& Weiser, 1975; 1985; Lipa, 1977), there has not been any other related with other Nosema species in G. mellonella. Nosema apis and Nosema ceranae are parasitic microsporidia that cause Nosemosis, an adult honey bee disease and infects all colony members i.e. the queen bee, worker bees and drones (Chen et al., 2009). Nosema infections are thought to be the main cause of honey bee winter losses in many countries (Higes et al., 2005; Higes et al., 2008; vanEngelsdorp \& Meixner, 2010). Although having a similar morphology under the light microscope, $N$. ceranae spores are slightly smaller and more circular than that of $N$. apis. The main differences between these two species was determined by electron microscop- ic structure, the small subunit (16S) rRNA gene sequences and ITS regions. Under an electron microscope, $N$. apis spores were observed to have 30 polar filaments while $N$. ceranae spores 20-23 polar filaments (Fries et al., 1996; 2006; Fries, 2010).

Nosema spp. can be vertically and horizontally transmitted in high quantities among honey bees (Higes et al., 2008). Although there are many factors in the spreading of Nosema, that Nosema spp. is known to mostly spread by transfer between organisms because of its host-switching property. It is necessary to investigate the probability of Nosema transmission between honey bees and other species which interact with them. The aim of this study was to investigate the presence of Nosema species in greater wax moth $G$. mellonella both collected from apiaries in the Isparta and Artvin provinces of Turkey and grown away from its natural habitat in laboratory conditions.

\section{MATERIAL AND METHODS}

\section{Microscopic examination of intestinal contents of Galleria mellonella larvae and adults}

In this study, the classical Nosema detection method applied to honey bees was used for G. mellonella samples (Fig. 1). Twenty homogeneous colonies of Apis mellifera anatoliaca and

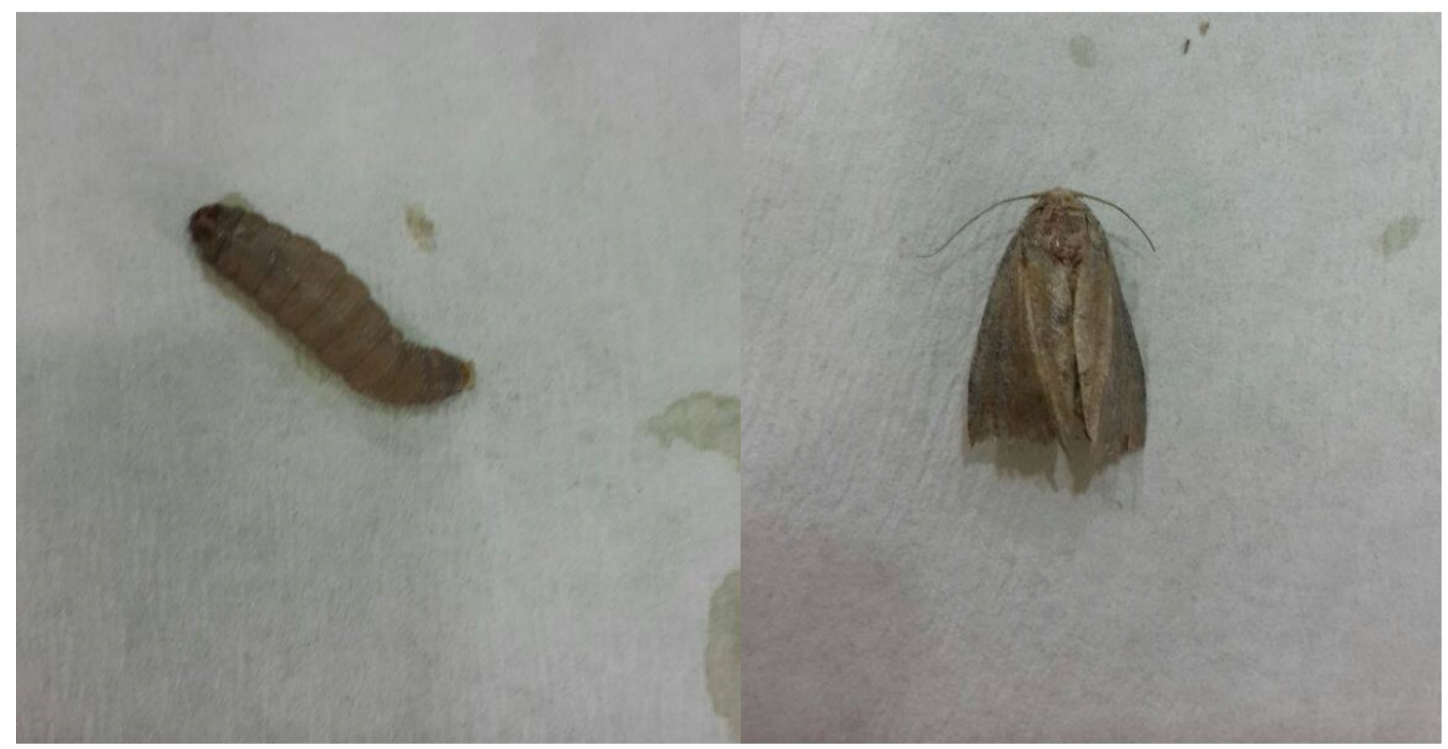

Fig. 1. Late instar larvae (left) and adult (right) phases of Galleria mellonella. 
Apis mellifera caucasica in the Isparta and Artvin provinces of Turkey were visited from January 2012 to April 2012 in order to find wax moth samples and adults and late instar larvae of $G$. mellonella samples were collected from wax moth-positive apiaries. All samples were stored at $-20^{\circ} \mathrm{C}$ until they were used for experiments. Adult and late instar larval samples of wax moth which were reared in the laboratory conditions were also selected in the same quantities for the study. All samples were washed first with $1 \%$ sodium hypochlorite solution and then with sterile distilled water to avoid any surface contamination and to determine whether they had Nosema spores in their gastrointestinal tracts. All materials used in this study were sterilized to prevent external contamination and new sterile materials were used before work with each sample. The solutions were prepared through homogenization of the intestinal content of fifteen greater wax moths per sample. Larva solutions were prepared by using the clean gut tissues of the larvae. The adult forms were homogenized to make adult solutions. Larva and adult samples were homogenized separately in order for these two stages of the wax moth to be compared. These solutions were centrifuged at 6,000 rpm for 10 minutes, and afterwards the supernatant was poured and the pellet was used for examination. $1 \mathrm{~mL}$ of distilled water was added per sample on the pellet and vortexed. These pellet solutions were used for the morphological detection of $N$. apis and $N$. ceranae spores with light microscope using an improved Neubauer slide (Cantwell, 1970).

\section{DNA isolation}

The Biospeedy DNA Isolation Kit (Bioeksen, Turkey) was used for DNA isolation. After homogenization of the moth samples, each was transferred to a microfuge tube weighing $200 \mathrm{mg}$. $400 \mu \mathrm{L}$ Guanidinium thiocyanate (0.1 MTris; $\mathrm{pH}$ 7.5) was added to the tube and vortexed at 3,000 rpm for one minute. The samples were incubated for $10 \mathrm{~min}$ at $95^{\circ} \mathrm{C}$. After stirring for $1 \mathrm{~min}$ at 3,000 rpm with vortex, the supernatant was centrifuged for $1 \mathrm{~min}$ at 14,000 rpm and then transferred to a new tube. $200 \mu \mathrm{L}$ of isopropanol was added to the supernatant and mixed thoroughly. The mixture was added to a DNA column followed by a 1-2 minute pause; after centrifugation at 12,000 rpm for 1 minute the pellet was discarded. $500 \mu \mathrm{L}$ Wash Buffer (20 mM NaCl, 2 mM Tris-HCl, pH 7.5; 80\% v/v Ethanol) was added to the column and pellet was discarded after centrifugation at 12,000 rpm for $1 \mathrm{~min}$. This step was repeated two more times. After the column was centrifuged for 1 min at 12,000 rpm, it was placed in the sterile micro centrifuge tube. $100 \mu \mathrm{L}$ solubilization buffer was added to the tube, incubated $1 \mathrm{~min}$ at room temperature and centrifuged at 14,000 rpm for 1 min, after which DNA isolates were obtained and stored at $-20^{\circ} \mathrm{C}$.

\section{Real-time PCR amplification (qPCR)}

After DNA isolation, real-time PCR amplification was performed through the modification of the protocol of Bourgeois et al. (2010). Biospeedy qPCR 2 × Master Mix (Bioeksen, Turkey) were used for this process. The ITS region has been targeted to distinguish Nosema species with the use of Nosema spp. specific forward primer ITS-F5'- TGAATGTGTCCCTGTTCTTTGTAC-3', N. apis specific reverse primer N.apisITS$\mathrm{R}$ 5'-TAATTATAATCTCCTTGGTCCGTG-3' and N.ceranae specific reverse primer NcerlTS-R TAAATATAATCTCCTGGTCGGTT. Biorad CFX Connect (Bio-Rad Laboratories, USA) was used in all reactions. The reaction contained $1.5 \mathrm{mM}$ $\mathrm{MgCl}_{2}, 0.2 \mathrm{mM}$ dNTP mix, $1 \times$ Reaction Buffer, $0.1 U$ Fast Start Taq DNA Polymerase, $1 \times$ EvaGreen, $4 \mathrm{ng} / \mu \mathrm{L}$ template DNA and $0.5 \mu \mathrm{M}$ of each primer. The optimization of specific primer pairs in the device was provided and thermocycling program given in Tab. 1 was applied. To determine whether there was a production of the expected product during qPCR, a melting curve analysis between $65^{\circ} \mathrm{C}-98^{\circ} \mathrm{C}$ was performed. qPCR data were analyzed with CFX Manager Software 3.0.

\section{RESULTS}

Microscopic examination showed that both $N$. apis and $N$. ceranae spores were found microscopically in all homogenates of G. mellonella 
Q-PCR thermocycling program designed for the molecular determination

Table 1 of Nosema apis and Nosema ceranae

\begin{tabular}{|c|c|c|c|c|}
\hline Detection Format & \multicolumn{4}{|c|}{ Reaction Volume } \\
\hline SYBR Green & \multicolumn{4}{|c|}{$20 \mu$} \\
\hline \multicolumn{5}{|l|}{ Programs } \\
\hline The Program Name & Number of cycles & Analysis Mode & & \\
\hline Pre-incubation & 1 & & & \\
\hline Ргoliferation & 45 & Counting & & \\
\hline Melting Curve & 1 & Melting Curve & & \\
\hline Cooling & 1 & & & \\
\hline \multicolumn{5}{|l|}{ Temperature Targets } \\
\hline Target $\left({ }^{\circ} \mathrm{C}\right)$ & Reading Mode & $\begin{array}{l}\text { R e t e n t i o n } \\
\text { (hh:mm:ss) }\end{array}$ & $\begin{array}{l}\text { Speed } \\
\left({ }^{\circ} \mathrm{C} / \mathrm{s}\right)\end{array}$ & $\begin{array}{r}\text { Reading } \\
\left(\text { Per }^{\circ} \mathrm{C}\right)\end{array}$ \\
\hline \multicolumn{5}{|l|}{ Pre-incubation } \\
\hline 95 & & 00:10:00 & 4,8 & - \\
\hline \multicolumn{5}{|l|}{ Proliferation } \\
\hline 95 & & 00:00:15 & 4,8 & - \\
\hline 53 & & 00:00:15 & 2,5 & - \\
\hline 72 & Single & 00:00:30 & 4,8 & - \\
\hline \multicolumn{5}{|l|}{ Melting Curve } \\
\hline 95 & & 00:00:05 & - & - \\
\hline 65 & & 00:01:00 & - & - \\
\hline 98 & Continuous & - & 0.5 & 10 \\
\hline \multicolumn{5}{|l|}{ Cooling } \\
\hline 40 & Single & 00:00:30 & 2,5 & - \\
\hline
\end{tabular}

Fig. 2. Microscopic image of Nosema spores in the intestine of Galleria mellonella larvae collected from apiaries. 

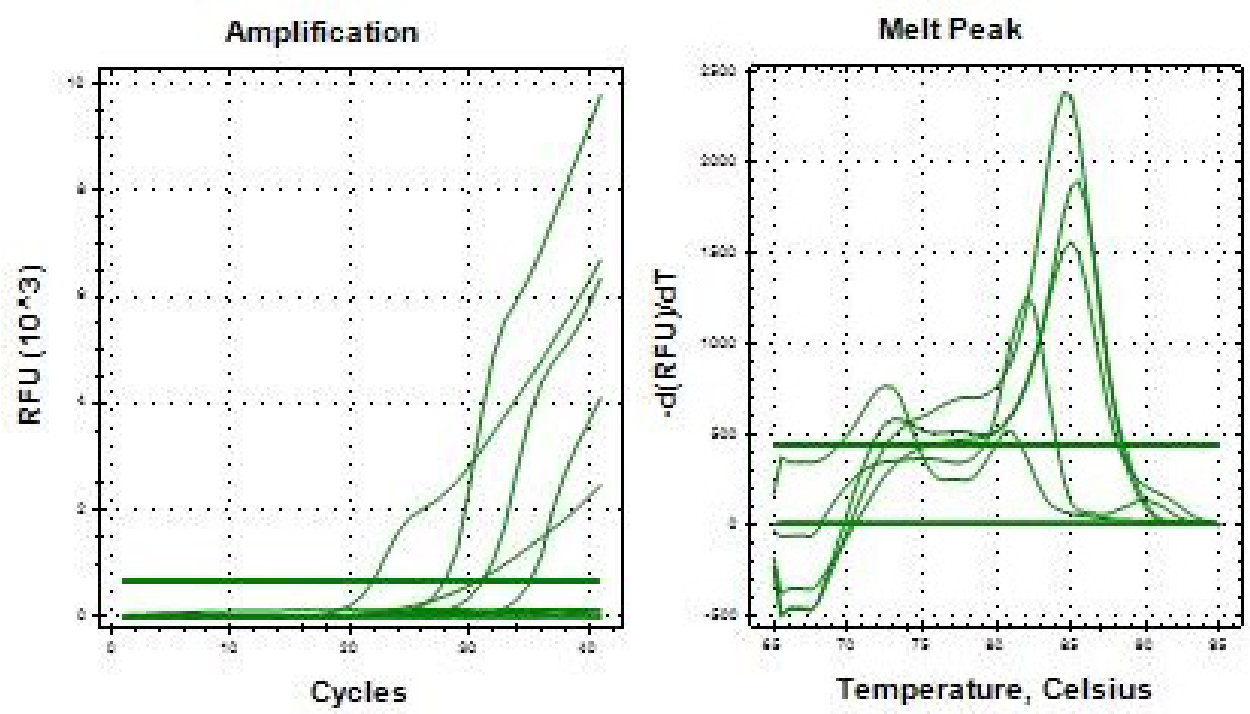

Fig. 3. Real-Time PCR proliferation threshold and melting curves obtained for Nosema ceranae and Nosema apis detection. Curves indicate positive results in terms of the presence of Nosema specie.

Table 2

Nosema species determination results depending on the melting temperature (Tm) and threshold cycle number (Ct) obtained from the qPCR results

\begin{tabular}{ccccc}
\hline Sample No & qPCR Target & Ct & Tm & Results \\
\hline 1 (Larvae) & N.apis & 31,14 & 85 & N.apis positive \\
1 (Larvae) & N.ceranae & 35,16 & 82 & N.ceranae positive \\
2 (Adult) & N.apis & 27,96 & 85 & N.apis positive \\
2 (Adult) & N.ceranae & - & - & N.ceranae negative \\
3 (Larvae) & N.apis & - & - & N.apis negative \\
3 (Larvae) & N.ceranae & 30,75 & 82 and 73 & N.ceranae positive \\
4 (Adult) & N.apis & 21,99 & 85 & N.apis positive \\
4 (Adult) & N.ceranae & - & - & N.ceranae negative \\
\hline
\end{tabular}

samples (Fig. 2). Although one Nosema species was detected in all sample groups, the two Nosema species were observed in only one sample group. Besides the Nosemaspores, some protozoan forms were seen in G. mellonella larvae and adult intestines.

Sensitivity of the detection assay was determined by amplification with real-time PCR, and these results' proliferation threshold and melting curves shown in Fig. 3. Determination of the presence of $N$. apis and $N$. ceranae using these results, cycle number (Ct) and melting temperature (Tm) data are given in Tab. 2. It was evaluated that qPCR was performed with
Ct $<35$ and positive results were obtained for $N$. ceranae at $\operatorname{Tm} 82^{\circ} \mathrm{C}$ and $N$. apis at $\operatorname{Tm} 85^{\circ} \mathrm{C}$.

The real-time PCR results of the Nosemaspecies were compared and $N$. apis was shown to be observed in wax moth adults which were both collected from apiary and reared in laboratory. The wax moth larvae reared in the laboratory only had $N$. ceranae while both Nosema species were found in larvae collected from the natural habitat (Tab. 2).

Comparison of the molecular and microscopic results revealed that consistent results were obtained in the two working process. Nosema 
species which were determined by the spore morphology were identical with the results obtained from real-time PCR studies.

\section{DISCUSSION}

N. ceranae, a significant pathogen for honey bees, shows a more lethal effect than $N$. apis. It is known to cause mass deaths and losses in honey bee colonies especially in strong colonies (Martín-Hernández et al., 2007; Higes et al., 2010). The spread and transfer of Nosema species to other organisms, especially $N$. ceranae, increases transmission to honey bees and wild bees. Therefore, the presence of Nosema species must be investigated in organisms which interact with honey bees. Real-time PCR studies showed that $N$. apis and $N$. ceranae were found in both the larvae and adult phases of G. mellonella, and thus Nosema species may be find in all stages. .

We began to obtain from the molecular samples results of between 20-35 cycles as a minimum relative fluorescence unit (RFU) peak-height level during Real-time amplification. Obtaining the results in lesser cycles showed that specific Nosema primers which targeted ITS regions were selected correctly for this study. The RFU peaks were also separated easily as a result of the sufficient amount of DNA being analyzed. RFU peak heights allowed us to achieve the true results more rapidly.

The results obtained from the Real-time PCR were directly proportional to the microscopic identification results of Nosema species. Samples identified as $N$. ceranae and $N$. apis during microscopic examination were also found to be identical with the results obtained from Real-time PCR. These results showed us that microscopic detection of Nosema species also provided reliable results in addition to molecular detection studies. However, studies only with light microscopy are not adequate for Nosema spore differentiation.

Many Nosema species are known to infect many species of invertebrates. There were discussions on each Nosema species being host-specific in various studies, but it has been shown in ex- periments that a Nosema species isolated from some Tribolium species infected many species including G. mellonella (Fisher \& Sanborn, 1962). $N$. ceranae originally found on Apis ceranae is now the parasite in $A$. mellifera and replacing $N$. apis which is known to infect honey bees for a long time. This situation shows that there is host-switching property in Nosema species and they can be transferred to the G. mellonella.

According to these results, Nosema species can be found in honey bee pests which may play a role in their transmission among honeybee colonies in addition to other transmission routes. These parasitic species may transfer from one honey bee hive to another with adult wax moths increasing the spread of parasitic Nosema species in honey bees. Furthermore, it is believed that Nosema spp. live a long time in $G$. mellonella without showing any symptoms because there was no change in the life quality of G. mellonella. The presence of Nosemaspecies in wax moths grown in laboratory conditions suggests to us that these organisms can be found in the normal microflora of wax moths. This situation will become clear after further research.

\section{REFERENCES}

Akyol, E., \& Korkmaz, A. (2008). The effect of $-5^{\circ} \mathrm{C}$ cold application to protect honeycombs against greater wax-moth Galleria mellonella damage. Uludag Bee Journal, 8(1), 26-29.

Bourgeois, A.L., Rinderer, T.E., Beaman, L.D., Danka, R.G. (2010). Genetic detection and quantification Nosema apis and $N$. ceranae in the honey bee. Journal of Invertebrate Pathology, 103(1), 53-58.

Cantwell, G.E. (1970). Standard methods for counting Nosema spores. American Bee Journal, 110,222-223.

Chen, P.Y., Evans, J.D., Murphy, C., Gutell, R., Zuker, M., Gundensen-Rindal, D., Pettis, J.S. (2009). Morphological, molecular and phylogenetic characterization of Nosemaceranae, a microsporidian parasite isolated from the European honey bee, Apis mellifera. Journal of Eukaryotic Microbiology, 56(2), 


\section{J. APIC. SCLI. VOL. G1 NOL. 22017}

\section{2-147. DOl: 10.1111/j.1550-7408.2008.00374.x}

Coleman, J.j., Muhammed, M., Kasperkovitz, P.V., Vyas, J.M., Mylonakis, E. (2011). Fusarium pathogenesis investigated using Galleria mellonella as a heterologous host. Fungal Biology, 715(12), 12791289. DOl: 10.1016/j.funbio.2011.09.005

Ellis, J.D., Graham, J.R., \& Mortensen, A. (2013). Standard methods for wax moth research. Journal of Apicultural Research, 52(1), 1-17. DOl: http://dx.doi. org/10.3896/IBRA.1.52.1.10

vanEngelsdorp, D., \& Meixner, M.D. (2010). A historical review of managed honey bee populations in Europe and the United States and the factors that may affect them. Journal of Invertebrate Pathology, 103, 80-95. DOl: 10.1016/j.jip.2009.06.011

Fisher, F.M., \& Sanborn, R.C. (1962). Observations on the Susceptibility of Some Insects to Nosema (Microsporidia: Sporozoa). The Journal of Parasitology, 48(6), 926-932. DOI: 10.2307/3275124

Fries, I., Feng, F., DaSilva, A., Slemenda, S.B., Pieniazek, N.J. (1996). Nosema ceranae n. sp. (Мicrospora, Nosematidae), morphological and molecular characterization of a microsporidian parasite of the Asian honey bee Apis cerana (Hymenoptera: Apidae). European Journal of Protistology, 32(3), 356-365.

Fries, I., Martin, R., Meana, A., García-Palencia, P." Higes, M. (2006). Natural infections of Nosema ceranae in European honey bees. Journal of Apicultural Research, 45(4), 230-233. D0l: 10.3896/ IBRA.1.45.4.13

Fries, I. (2010). Nosema ceranae in European honey bees (Apis mellifera). Journal of Invertebrate Pathology, 103, 573-579. DOl: 10.1016/j. jip.2009.06.017

Harding, C.R., Schroeder, G.N., Collins, I.W., Frankel, G. (2013). Use of Galleria mellonellaas a Model Organism to Study Legionella pneumophila Infection. Journal of Visualized Experiments, 81, 1-10.

Higes, M., Martín, R., Sanz, A., Alvarez, N., Sanz,
A., Garcia, M.P., Meana, A. (2005). El síndrome de despoblamiento de las colmenas en Espaňa. Consideraciones sobre su origen. Vida Apícola, 133, $15-21$.

Higes, M., Martin-Hernandez, H., Botias, C., Bailon, E.G., Gonzalez-Porto, A.V., Barrios, L... Meana, A (2008). How natural infection by Nosema ceranae causes honeybee colony collapse. Environmental Microbiology, 1910),2659-2669.DOl: 10.1111/j.14622920.2008.01687.x

Higes, M., Martín-Hernández, R., \& Meana, A. (2010). Nosema ceranae in Europe: an emergent type C Nosemosis. Apidology, 47(3), 375-392. DOl: https:// doi.org/10.1051/apido/2010019

Hu, K., \& Webster, J.M. (2000). Antibiotic production in relation to bacterial growth and nematode development in Photorhabdus-Heterorhabditis infected Galleria mellonellalarvae. FEMS Microbiology Letters, 189(2), 219-23.

Kučera, M., \& Weiser,.).(1975). Lactate dehydrogenase isoenzymes in the larvae of Barathra brassicae and Galleria mellonella during microsporidan infection. Journal of Invertebrate Pathology, 25(1), 109-114.

Kučera, M., \& Weiser, J. (1985). Different course of proteolytic inhibitory activity and proteolytic activity in Galleria mellonella larvae infected by Nosema algerae and Vairimorpha heterosporum. Journal of Invertebrate Pathology, 45(1), 41-46.

Lipa, ... (1977). Microsporidian infections of Galleria mellonella (L.) (Lepidoptera, Galleriidae) with the description of a new species Nosema galleriae n.sp. Acta Protozoologica, 16(2), 141-150.

Martín-Hernández, R., Meana, A., Prieto, L., Martínez Salvador, A., Garrido-Bailón, E., Higes, M. (2007). Outcome of colonization of Apis mellifera by Nosema ceranae. Applied and Environmental Microbiology, 73(20), 6331-6338. DOI: 10.1128/AEM.00270-07

Spangler, H.G. (1985). Sound production and communication by the greater wax moth (Lepidoptera: Pyralidae). Annals of the Entomological Society of America, 78(1), 54-61. 


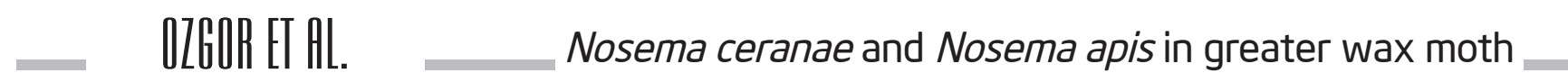

Williams, J.L. (1997). Insects: Lepidoptera (moths). In R Morse; K Flottum (Eds). Honey bee pests, predators, and diseases (pp. 121-141). Ohio, USA: The Al Root Company. 